\title{
Éditorial
}

\section{Argent public, argent privé : une nécessaire synergie.}

\author{
Pierre Moszkowicz* \\ Rédacteur en chef, Insa de Lyon
}

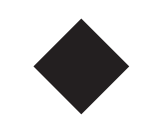

Le CNRS a inscrit dans son contrat d'action pluriannuel 2002-2005 cinq axes prioritaires, dont l'un concerne l'Environnement, l'Energie et le Développement Durable.

Un objectif affiché par le CNRS est en particulier de devenir un acteur de premier plan de la recherche en Environnement et Energie en Europe et dans le monde. Pour cela il faut inciter les laboratoires à s'investir dans une recherche par essence interdisciplinaire, en relation avec les autres organismes de recherche et les industriels. Un colloque a réuni en décembre environ 400 chercheurs du CNRS et des grands organismes concernés (INRA, BRGM, CEMAGREF, CIRAD, CNES, IRD, LCPC, IFREMER...) pour établir la synthèse des travaux de prospective qui se sont déroulés pendant l'année écoulée (http://www.insu.cnrsdir.fr/pdf/prospective_sic.pdf).

Une programmation de la recherche sera pilotée par l'INSU (Institut National des Sciences de l'Univers) qui devient I'INSUE (E comme environnement). Cet institut transversal par rapport aux diverses directions du CNRS (Sciences de l'Univers, Sciences de la Vie, Sciences Humaines et Sociales, Sciences pour l'Ingénieur, Sciences Chimiques...) aura comme mission de structurer des actions collectives de recherche et d'affecter les moyens nécessaires à leur mise en œuvre. La mobilisation des chercheurs est aujour- d'hui forte pour s'engager hors des « sentiers battus » de leurs champs disciplinaires respectifs et très nombreux sont les sujets où pourraient jouer à plein les interactions entre la recherche fondamentale et la recherche appliquée.

Une faiblesse de la recherche française apparaît cependant encore très nette : les relations entre recherche publique et recherche industrielle privée ne sont pas suffisantes, surtout dans des domaines tels que l'environnement, où les enjeux industriels et sociétaux sont très directement liés aux résultats de la recherche. La part de la dépense intérieure de recherche et développement (DIRD) par rapport au produit intérieur brut (PIB) n'est ainsi que de $2,2 \%$, ce qui place la France loin derrière les USA (2,74 \%) ou le Japon (3\%). Encore plus inquiétant, le ratio DIRD/PIB a régulièrement diminué depuis 1993 (il était égal à 2,45\%) et ce recul s'explique en grande partie par la baisse des budgets privés de la recherche industrielle.

L'affichage d'objectifs ambitieux pour une recherche au service de la société et la mise en œuvre à cet effet d'importants moyens devront permettre d'améliorer la synergie entre financements publics et financements privés. Ce sera certainement l'une des clés du succès des projets du CNRS.

\section{*Pierre Moszkowicz}

Directeur du LAEPSI, Laboratoire d'analyse environnementale des procédés et des systèmes Industriels - INSA de Lyon - moszkowicz@insa-lyon.fr 